\title{
Erratum to: Altered functional connectivity networks in acallosal and socially impaired BTBR mice
}

\author{
Francesco Sforazzini - Alice Berterto $\cdot$ Luca Dodero • \\ Gergely David · Alberto Galbusera • Angelo Bifone • \\ Maria Luisa Scattoni $\cdot$ Massimo Pasqualetti $\cdot$ Alessandro Gozzi
}

Published online: 27 December 2014

(c) Springer-Verlag Berlin Heidelberg 2014

\section{Erratum to: Brain Struct Funct \\ DOI 10.1007/s00429-014-0948-9}

Unfortunately, the published author group omitted the contribution of Angelo Bifone. The correct and complete author group is given below:

Francesco Sforazzini $^{1}$, Alice Berterto ${ }^{1,2}$, Luca Dodero ${ }^{1,3}$, Gergely David $^{1}$, Alberto Galbusera ${ }^{1}$, Angelo Bifone ${ }^{1}$, Maria Luisa Scattoni ${ }^{4}$, Massimo Pasqualetti ${ }^{2}$, Alessandro Gozzi ${ }^{1}$

${ }^{1}$ Center for Neuroscience and Cognitive Systems @ UniTn, Istituto Italiano di Tecnologia, 38068 Rovereto, Italy

${ }^{2}$ Department of Biology, Unit of Cell and Developmental Biology, University of Pisa, Pisa, Italy

${ }^{3}$ Istituto Italiano di Tecnologia, Pavis Lab, Genoa, Italy

The online version of the original article can be found under doi:10.1007/s00429-014-0948-9.

F. Sforazzini · A. Berterto - L. Dodero · G. David .

A. Galbusera · A. Bifone · A. Gozzi ( $\square)$

Center for Neuroscience and Cognitive Systems @ UniTn,

Istituto Italiano di Tecnologia, 38068 Rovereto, Italy

e-mail: alessandro.gozzi@iit.it

A. Berterto $\cdot$ M. Pasqualetti

Department of Biology, Unit of Cell and Developmental

Biology, University of Pisa, Pisa, Italy

L. Dodero

Istituto Italiano di Tecnologia, Pavis Lab, Genoa, Italy

\section{L. Scattoni}

Neurotoxicology and Neuroendocrinology Section, Department of Cell Biology and Neurosciences, Istituto Superiore di Sanità, Rome, Italy
${ }^{4}$ Neurotoxicology and Neuroendocrinology Section, Department of Cell Biology and Neurosciences, Istituto Superiore di Sanità, Rome, Italy 